\title{
Nanoscale Investigation of Frost Formation on Cold Plates
}

\author{
Kentaro Nagashima ${ }^{1}$, Mitsuhiro Matsumoto ${ }^{1}$ \\ ${ }^{1}$ Department of Mechanical Engineering and Science, Kyoto University \\ Kyoto-Daigaku-Katsura, Kyoto 615-8540, Japan \\ nagashima.kentarou.55w@st.kyoto-u.ac.jp; matsumoto@kues.kyoto-u.ac.jp
}

\begin{abstract}
We investigated the atomic scale mechanism of frost formation on a cold plate with molecular dynamics (MD) simulations. The frost formation is a special type of heterogeneous nucleation process, where supersaturated vapor becomes condensed on the sufficiently cold surface without passing through liquid state (desublimation). Thus we expect this only for a narrow range of conditions (temperature and vapor density) and it may take substantially long time on the atomic scale. We conducted a series of MD simulations with two different types of water model, i.e., TIP4P full-atom model and $\mathrm{mW}$ coarse-grained one. For very long simulations with the $\mathrm{mW}$, we successfully observed the formation of bilayer hexagonal ice on plates of 100-200 K.
\end{abstract}

Keywords: water, frost formation, desublimation, molecular dynamics simulation

\section{Introduction}

Standard heat exchangers in the condenser units of air conditioners have a serious problem in winter; thick frost is formed on the surface, which deteriorates the thermal efficiency. Much effort has been devoted to experimental investigations and theoretical modeling, but there still exist many questions about the microscopic process of the frost formation.

Frost is generally an assembly of ice microcrystals. As water vapor becomes contacted with sufficiently cold plates, the vapor temperature decreases below the freezing point and get "condensed" without passing through a liquid state (desublimation). This is a type of surface induced (i.e., heterogenous) nucleation, and should have several relevant control parameters, such as the plate temperature, vapor temperature, vapor density, surface wettability, and the pre-existing nuclei on the surface. To develop "frost-free" heat exchangers, understanding the mechanism in terms of these parameters is essential.

The purpose of this study is to elucidate how frost starts to form on various solid surface on atomic scales. Since the frost formation is a nucleation process, this should be stochastic and will generally take long time on atomic scales. Lower plate temperature and higher vapor density may accelerate the process, but too large condensing speed will lead to amorphous states. Thus we expect that the frost formation occurs only in some narrow range of control parameters. In this work, we adopt two models for water molecules; a full atom model (TIP4P/2005), which is one of the standard models for water simulation, and a recently developed coarse grained model $(\mathrm{mW})$, which reduces the computational cost and enables us to carry out long simulations.

\section{Simulation Method}

The LAMMPS code was used for the molecular dynamics simulation [1]. The target system of $20 \mathrm{~nm} \times 20 \mathrm{~nm} \times 50 \mathrm{~nm}$ consists of a solid wall and a gas phase region. The solid wall is assumed to be a platinum fcc crystal with Lennard-Jones (LJ) atomic interaction [2]. Water vapor mixed with carrier gas (argon) is introduced in the gas phase region. The TIP4P/2005 rigid rotor model [3] and $\mathrm{mW} \mathrm{[4]} \mathrm{are} \mathrm{adopted} \mathrm{for} \mathrm{water} \mathrm{molecules,} \mathrm{and} \mathrm{the} \mathrm{LJ} \mathrm{(12-6)} \mathrm{potential} \mathrm{is} \mathrm{assumed} \mathrm{for} \mathrm{the} \mathrm{argon.} \mathrm{The}$ conventional Lorentz-Berthelot rule is applied.

Periodic boundary conditions are assumed for the horizontal directions, while a reflective boundary is utilized by introducing an LJ wall on the ceiling. The substrate (Pt crystal) consists of four layers; atomic positions are fixed for the bottom two layers to prevent the translational motion of the substrate during the deposition. Pt atoms in the top two layers are temperature-controlled with the Nosé-Hoover thermostat algorithm. The energy parameter $\varepsilon$ for the Pt is adjusted so that only the central part of the substrate is hydrophilic; water molecules can condense only on this area. 
After equilibrating the entire system (except for the fixed bottom layers) at $T=300 \mathrm{~K}$, the temperature control is continued for the carrier gas $(\mathrm{Ar})$ at $300 \mathrm{~K}$ and the substrate at $T_{s}$; we choose several values for $T_{s}$, from $100 \mathrm{~K}$ to $250 \mathrm{~K}$, to see how the substrate temperature affects the frost formation. So far we have done the simulation with single condition of supersaturation (the ratio of water vapor density to the saturated density at $300 \mathrm{~K}$ ), but the supersaturation dependence is to be investigated. The relevant conditions are described in Table 1.

Table 1: Simulation conditions

\begin{tabular}{c|c|c|c|c}
\hline & Case 1 & Case 2 & Case 3 & Case 4 \\
\hline Surface temperature $T_{\mathrm{s}}[\mathrm{K}]$ & 100 & 150 & 200 & 250 \\
\hline Supersaturation ratio (at $300 \mathrm{~K})$ & 32.7 & 32.7 & 32.7 & 32.7 \\
\hline
\end{tabular}

\section{Results/Discussion}

An example of vapor condensation on the substrate is shown in Fig. 1. Water molecules are adsorbed on the hydrophilic region to form a condensate. The shape becomes more flattened for lower $T_{\mathrm{s}}$.

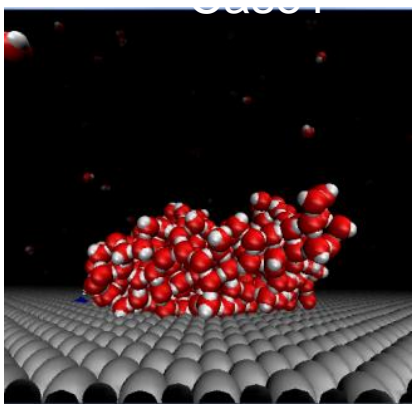

\subsection{TIP4P/2005 model}

The TIP4P/2005 rigid rotor model is a standard water model and widely used to investigate properties of ices and low-temperature liquid [3]. Since each molecule has four interaction sites and the long-ranged Coulombic force should be properly treated, computational costs are fairly large.

The number of condensed water molecules is plotted against time in Fig. 2, which indicates that the size of the condensate hardly depends on the substrate temperature, except for Case 4. The thickness (height) of the condensate in $5 \mathrm{~ns}$ is shown in Fig. 3, which indicates the thickness increases as the temperature rises. The condensate tends to take a spherical shape due to the surface tension; at extremely low $T_{\mathrm{s}}$, however, condensed water molecules freeze immediately and cannot move, leading to the flattened amorphous structure. For the lowest $T_{\mathrm{s}}$ (Case 1), the condensate has a distorted shape, as seen in Fig. 1.

To see the structure of the condensate, the oxygen-oxygen radial distribution function (RDF) is evaluated (Fig. 4), which indicates disordered structure for all cases. We preliminarily conclude that amorphous ice is formed in Cases 1 and 2, while the condensate is a supercooled liquid droplet in Cases 3 and 4. 


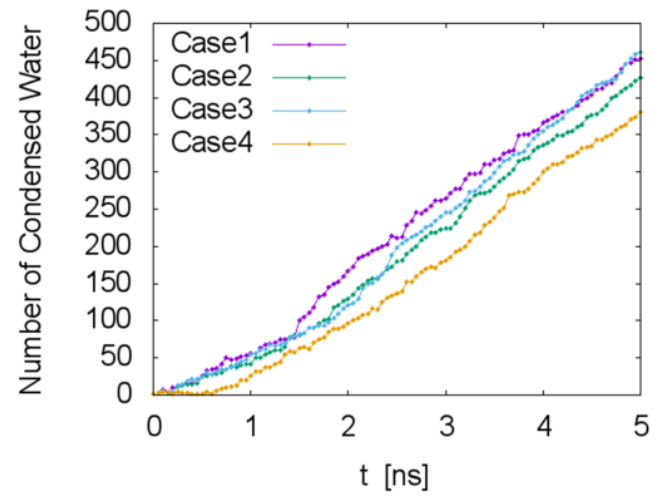

Figure 2: Size of the condensate (TIP4P/2005).

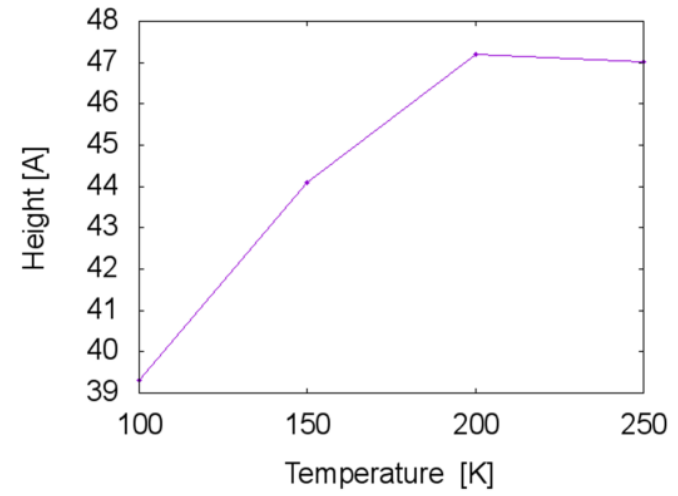

Figure 3: Height of the condensate at $5 \mathrm{~ns}(\mathrm{TIP} 4 \mathrm{P} / 2005)$.

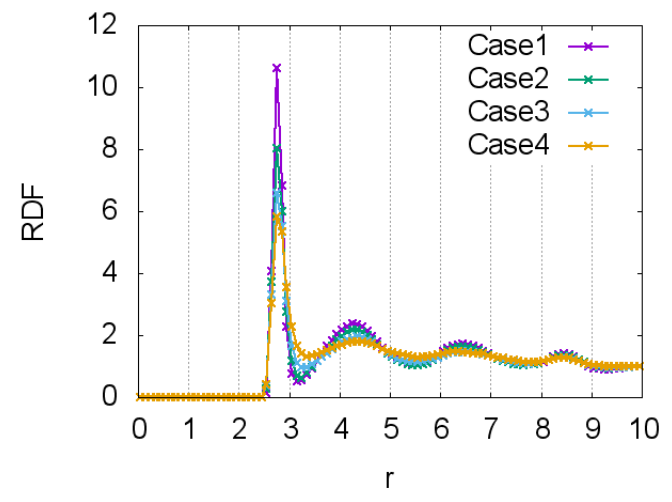

Figure 4: O-O radial distribution function of adsorbed water molecules (TIP4P/2005).

\section{2. $\mathrm{mW}$ Model}

The recently proposed $\mathrm{mW}$ potential [4] is a coarse-grained single site model with silicon-like three body interactions; two-dimensional ice growth on a substrate was successfully investigated with this model [5]. The computational cost is much lower than TIP4P/2005 model since the potential has no Coulombic interactions and there is no need to trace the rotational degrees of freedom. Thus we can carry out longer MD simulations under relatively moderate conditions.

We compare the two models; the oxygen-oxygen RDF is shown in Fig. 5 and the angle distribution of neighbouring O$\mathrm{O}-\mathrm{O}$ in Fig. 6. The shape of the first peak in RDF is almost the same, but the second peak in the $\mathrm{mW}$ is sharper than that of

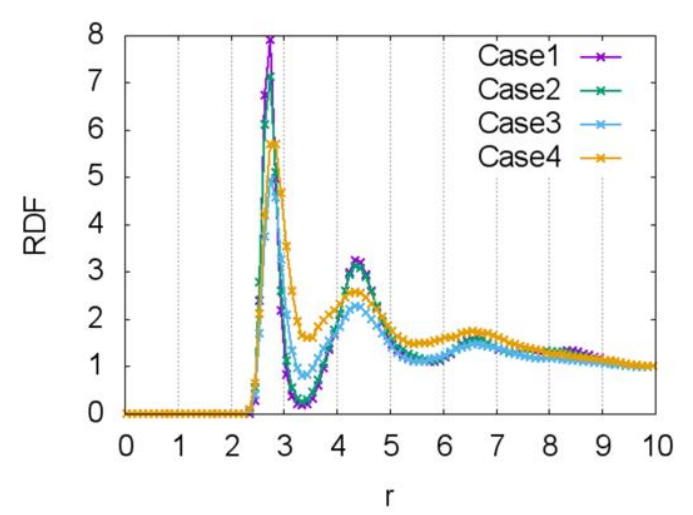

Figure 5: O-O radial distribution function of adsorbed water molecules $(\mathrm{mW})$.

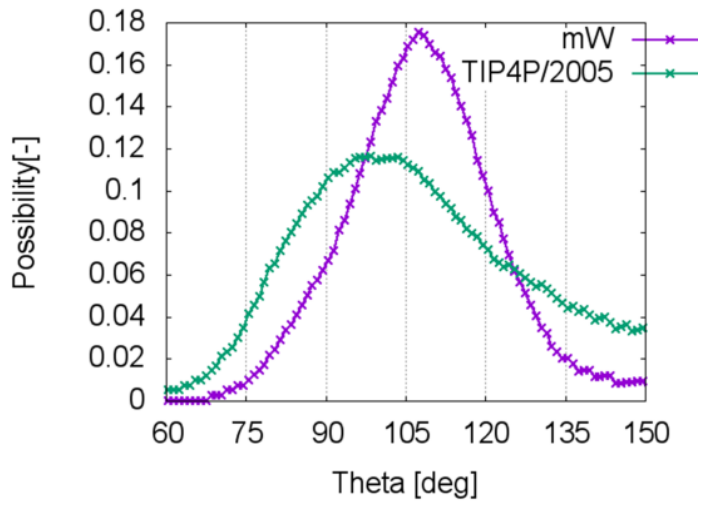

Figure 6: O-O-O angular distribution of adsorbed water molecules (Case 1). 
the TIP4P/2005. For the angle distribution, the $\mathrm{mW}$ model has a peak at $107.5^{\circ}$ while the TIP4P/2005 has a peak at $98.5^{\circ}$. Based on these observations, we conclude that the $\mathrm{mW}$ model gives a more crystal-like condensate.

\subsection{ICE Formation on Larger Area ( $\mathrm{mW}$ )}

So far we have limited the adsorption (i.e., hydrophilic) area to a circular region of radius $1.0 \mathrm{~nm}$; note that the size of the whole plate is $20 \mathrm{~nm} \times 20 \mathrm{~nm}$. To increase the nucleation probability, we increased the area to radius $8.0 \mathrm{~nm}$ and performed a simulation using the $\mathrm{mW}$ model.

For the highest $T_{\mathrm{s}}$ case (Case 4), a supercooled liquid droplet is observed, as shown in Fig. 7. In Cases 1, 2, and 3, however, bilayer hexagonal ice crystal is observed. During the growth of bilayer hexagonal ice, impinged water molecules do not pile up on the bilayer ice but tend to adhere to the periphery.

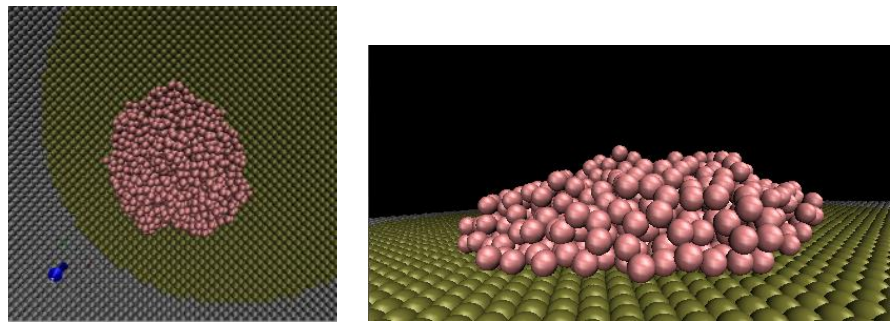

Figure 7: Snapshots at $20 \mathrm{~ns}$ for Case4 (mW).
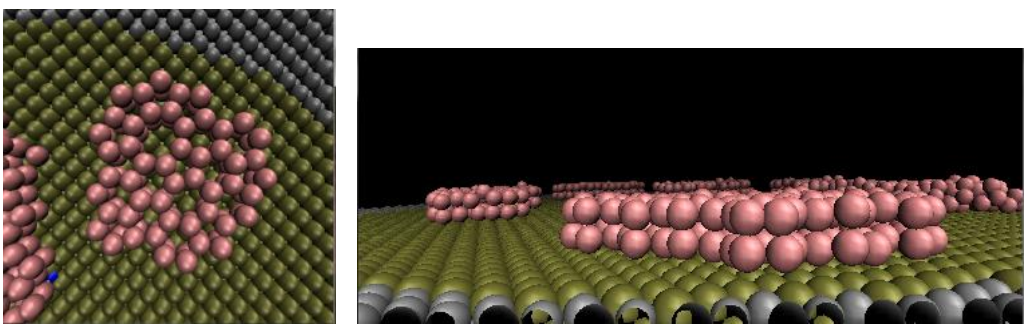

Figure 8: Snapshots at $20 \mathrm{~ns}$ for Case1 $(\mathrm{mW})$.

\section{Conclusion}

The process of frost formation from supersaturated water vapor was investigated using MD simulation. Growth from vapor to amorphous ice and supercooled water was observed using two models, a conventional TIP4P/2005, and a coarse-grained $\mathrm{mW}$. The $\mathrm{mW}$ seems more suitable to simulate the desublimation. In the system with larger hydrophilic region, the growth of bilayer hexagonal ice was observed at lower surface temperature. Investigation of other parameters, such as wettability, is under way.

\section{References}

[1] https://lammps.sandia.gov/

[2] D. Toghraie, M. Mokhtari, M. Afrand, "Molecular dynamic simulation of copper and platinum nanoparticles Poiseuille flow in a nanochannels," Physica E, 84 (2016) 152.

[3] J. L. F. Abascal, C. Vega, "A general purpose model for the condensed phases of water: TIP4P/2005," J. Chem. Phys., 123 (2005) 234505.

[4] V. Molinero, E. B. Moore, "Water modeled as an intermediate element between carbon and silicon", J. Chem. Phys., B, 113 (2009) 4008-4016.

[5] R. Ma, D. Cao, C. Zhu, Y. Tian, J. Peng, J. Guo, J. Chen, X-Z. Li, J. S. Francisco, X. C. Zeng, L-M. Xu, E-G. Wang and Y. Jiang, "Atomic imaging of the edge structure and growth of a two-dimensional hexagonal ice," Nature, 577 (2020) pp. 60-63. 